\title{
Flagella by numbers: comparative genomic analysis of the supernumerary flagellar systems among the Enterobacterales
}

\author{
Pieter De Maayer ${ }^{1 *}$ (D), Talia Pillay ${ }^{1}$ and Teresa A. Coutinho ${ }^{2}$
}

\begin{abstract}
Background: Flagellar motility is an efficient means of movement that allows bacteria to successfully colonize and compete with other microorganisms within their respective environments. The production and functioning of flagella is highly energy intensive and therefore flagellar motility is a tightly regulated process. Despite this, some bacteria have been observed to possess multiple flagellar systems which allow distinct forms of motility.

Results: Comparative genomic analyses showed that, in addition to the previously identified primary peritrichous (flag-1) and secondary, lateral (flag-2) flagellar loci, three novel types of flagellar loci, varying in both gene content and gene order, are encoded on the genomes of members of the order Enterobacterales. The flag-3 and flag-4 loci encode predicted peritrichous flagellar systems while the flag-5 locus encodes a polar flagellum. In total, 798/4028 $(\sim 20 \%)$ of the studied taxa incorporate dual flagellar systems, while nineteen taxa incorporate three distinct flagellar loci. Phylogenetic analyses indicate the complex evolutionary histories of the flagellar systems among the Enterobacterales.

Conclusions: Supernumerary flagellar loci are relatively common features across a broad taxonomic spectrum in the order Enterobacterales. Here, we report the occurrence of five (flag-1 to flag-5) flagellar loci on the genomes of enterobacterial taxa, as well as the occurrence of three flagellar systems in select members of the Enterobacterales. Considering the energetic burden of maintaining and operating multiple flagellar systems, they are likely to play a role in the ecological success of members of this family and we postulate on their potential biological functions.
\end{abstract}

Keywords: Enterobacterales, Supernumerary flagella, Flag, Evolution, Motility

\section{Background}

Flagella are complex structures that provide bacteria with an effective means of carrying out swimming (movement of single bacterial cells in liquid environments) and swarming (coordinated mobility of bacterial population on semi-solid or solid surfaces) movements [1]. Furthermore, they participate in biofilm formation,

\footnotetext{
* Correspondence: Pieter.Demaayer@wits.ac.za

'School of Molecular \& Cell Biology, University of the Witwatersrand, Wits 2050, South Africa

Full list of author information is available at the end of the article
}

adhesion to surfaces and host cell invasion [2-4]. As such, flagella provide bacteria with a considerable competitive advantage over non-motile microorganisms occupying the same ecological niche and $>80 \%$ of known bacterial species are known to produce and maintain these structures $[2,3]$.

The basic structure of the flagellum is relatively well conserved among the flagellate bacteria, comprising of a basal body, hook and flagellar filament [5]. However, extensive diversity occurs within and between bacterial lineages in terms of the number of flagella per cell, the positions of the flagella on the cell surface, the number of genes required for the production and regulation of

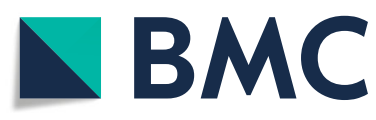

(c) The Author(s). 2020 Open Access This article is licensed under a Creative Commons Attribution 4.0 International License, which permits use, sharing, adaptation, distribution and reproduction in any medium or format, as long as you give appropriate credit to the original author(s) and the source, provide a link to the Creative Commons licence, and indicate if changes were made. The images or other third party material in this article are included in the article's Creative Commons licence, unless indicated otherwise in a credit line to the material. If material is not included in the article's Creative Commons licence and your intended use is not permitted by statutory regulation or exceeds the permitted use, you will need to obtain permission directly from the copyright holder. To view a copy of this licence, visit http://creativecommons.org/licenses/by/4.0/ The Creative Commons Public Domain Dedication waiver (http://creativecommons.org/publicdomain/zero/1.0/) applies to the data made available in this article, unless otherwise stated in a credit line to the data. 
flagella $[5,6]$. Bacterial flagellar arrangements vary from one polar flagellum (monotrichous), two polar flagella one at each end of the bacterial cell (amphitrichous), many polar flagella at one or both ends of the bacterial cell (lophotrichous), or several flagella distributed along the bacterial cell surface (peritrichous) [5]. In addition, a limited number of bacterial taxa produce dual flagellar systems encoded by distinct sets of genes present on the genome. This trait has been most widely studied in Vibrio and Aeromonas spp., which possess both a polar flagellum as well as several lateral (peritrichous) flagella [2, 6-9]. Dual flagellar systems have furthermore been observed in Azospirillum, Rhodospirillum and Helicobacter spp. [2]. The polar and lateral flagella allow these bacterial cells to carry out different modes of motility, where the polar flagellum facilitates swimming motility and the lateral flagella enable swarming motility [2, 69]. While this dual flagellar motility may provide the bacteria with a competitive advantage, flagellar synthesis and functioning is an energetically expensive commitment $[3,9]$. As such, bacteria that are capable of producing dual flagellar systems tightly regulate the production of polar and lateral flagella $[9,10]$.

Members of the order Enterobacterales are commonly isolated from a wide range of environments including air, soil and water and include some of the most wellknown pathogens of both plant and animal hosts [11, 12]. The ecological success of the Enterobacterales can in part be attributed to their capacity for motility, which is facilitated by their primary, peritrichous flagellar system $[12,13]$. In the enterobacterial model organisms Escherichia coli and Salmonella enterica, up to 50 genes are required the assembly, maintenance and functioning of this flagellum. These genes are organized in three genomic clusters, which are collectively termed the primary flagellar (flag-1) locus [3, 12, 13]. In addition to the flag-1 system, a second, evolutionary distinct, flagellar system, encoded by the flag-2 locus, was observed and shown to be relatively prevalent among members of the order Enterobacterales [14, 15]. The flag-2 locus encodes a lateral flagellar system and has been postulated to facilitate swarming motility among the Enterobacterales, although it has been inactivated through gene deletions and transposon integration in a substantial proportion of enterobacterial taxa [14-16]. Here, by means of a comprehensive comparative genomic analysis, we identified three additional distinct flagellar loci flag-3, flag-4 and flag-5 which are distributed among the Enterobacterales. These occur predominantly as a secondary flagellar system in enterobacterial taxa with the flag-1 primary flagellar system, while a limited subset of taxa incorporate three flagellar loci. Here we postulate on the evolutionary histories and potential functions of these supernumerary flagellar systems among the Enterobacterales.

\section{Results}

The Enterobacterales encode five distinct flagellar systems

The complete and draft genomes of 4082 taxa belonging to the order Enterobacterales were screened for the presence of additional flagellar (flag) loci by performing tBlastN analysis of the full complement of complete protein sequences required for the synthesis of the flag-1 and flag-2 flagella of E. coli K-12 strain MG1655 (47 proteins) and E. coli 042 (38 proteins), respectively, against the genome sequences. In total, $816(20.26 \%)$ of the studied strains incorporate at least two distinct flag loci (Table 1; Additional file 1: Table S1). When considering that 664 strains lack any flag loci altogether and can be considered as incapable of swimming motility, dual or multiple flag loci are hallmarks of $24.26 \%$ of the presumed motile enterobacterial taxa. The secondary flag loci are predominated by the previously characterized flag-2 loci, with 593 (575 strains with and 18 strains lacking flag-1 loci) of the studied strains incorporating this locus (Table 1) [15]. The protein complements encoded by the remaining additional flagellar loci, along with taxonomically representative flag- 1 and flag- 2 datasets were compared. A total of twenty-five distinct single-copy orthologues (SCOs) are conserved among the loci and were aligned and concatenated to generate a Maximum Likelihood (ML) phylogeny. The resultant SCO phylogeny showed that, aside from the flag-1 and flag-2 loci, the remaining loci form three distinct clades, which we have termed flag-3, flag-4 and flag-5 loci (Fig. 1). The largest clade incorporates the novel flag-3 loci, which occur in 249 distinct taxa $(6.18 \%$ of total taxa studied), across a broad taxonomic spectrum, including members of the families Enterobacteriaceae Erwiniaceae, Hafniaceae, Morganellaceae and Yersiniaceae (Table 1; Fig. 2). By contrast, the flag-4 and flag-5 loci are less prevalent, being restricted to six (four Pectobacteriaceae and two Yersiniaceae members) and eight taxa (all Plesiomonas shigelloides isolates; Family unassigned), respectively (Table 1; Fig. 2).

The flag-3 loci are predominantly found in enterobacteria that also harbour a flag-1 locus, with only Rahnella variigena DLL 7529 incorporating a flag-3 locus, but lacking the flag-1 system. This is similar to the flag-2 system which, with the exception of ten taxa, occur in flag-1 flagellated taxa [15]. The flag-4 system occurs in three taxa (Sodalis spp.) that also incorporate flag-1 loci, while the other three (two Wigglesworthia spp. and Biostraticola tofi DSM 19580) lack the latter locus. All flag-5 encoding $P$. shigelloides strains also incorporate a flag-2 locus, but lack a flag-1 locus. While the majority of enterobacteria with supernumerary flagellar loci incorporate two flag loci in their genome, nineteen taxa ( $0.5 \%$ of all strains studied) incorporate three distinct flag 
Table 1 Prevalence of the primary (flag-1) and additional (flag-2 to flag-5) loci among the Enterobacterales. The number and percentage of taxa at the family and genus level that incorporate each type of locus are indicated

\begin{tabular}{|c|c|c|c|c|c|c|c|c|c|}
\hline Family & Genus & \# Strains & flag-1 & flag-2 & flag-3a & flag-3b & flag-4 & flag-5 & $\begin{array}{l}\text { \# Strains with } \\
3 \text { flag loci }\end{array}$ \\
\hline \multirow[t]{5}{*}{ Budviciaceae } & & 9 & $0(0 \%)$ & 7 (77.8\%) & $0(0 \%)$ & $0(0 \%)$ & $0(0 \%)$ & $0(0 \%)$ & \\
\hline & Budvicia & 2 & - & $2(100 \%)$ & - & - & - & - & \\
\hline & Leminorella & 2 & - & $1(50 \%)$ & - & - & - & - & \\
\hline & Limnobaculum & 1 & - & - & - & - & - & - & \\
\hline & Pragia & 4 & - & $4(100 \%)$ & - & - & - & - & \\
\hline \multirow[t]{32}{*}{ Enterobacteriaceae } & & 2464 & $\begin{array}{c}1878 \\
(76.2 \%)\end{array}$ & $\begin{array}{c}317 \\
(12.9 \%)\end{array}$ & $\begin{array}{c}110 \\
(4.5 \%)\end{array}$ & $\begin{array}{c}34 \\
(1.4 \%)\end{array}$ & $\begin{array}{c}0 \\
(0 \%)\end{array}$ & $\begin{array}{c}0 \\
(0 \%)\end{array}$ & $\begin{array}{c}7 \\
(0.3 \%)\end{array}$ \\
\hline & Atlantibacter & 5 & $5(100 \%)$ & - & - & - & - & - & \\
\hline & Buttiauxella & 8 & $8(100 \%)$ & $4(50 \%)$ & - & $1(12.5 \%)$ & - & - & $1(12.5 \%)$ \\
\hline & Cedecea & 8 & $8(100 \%)$ & $6(75 \%)$ & - & - & - & - & \\
\hline & Citrobacter Clade A & 157 & $157(100 \%)$ & $91(58.0 \%)$ & - & - & - & - & \\
\hline & Citrobacter Clade B & 21 & $21(100 \%)$ & $5(23.8 \%)$ & - & - & - & - & \\
\hline & Citrobacter Clade C & 4 & $4(100 \%)$ & $4(100 \%)$ & - & $4(100 \%)$ & - & - & $4(100 \%)$ \\
\hline & Citrobacter Clade D & 30 & $30(100 \%)$ & $30(100 \%)$ & - & - & - & - & \\
\hline & Cronobacter & 189 & $189(100 \%)$ & - & - & - & - & - & \\
\hline & Enterobacter & 608 & $608(100 \%)$ & $30(4.9 \%)$ & $\begin{array}{c}110 \\
(18.1 \%)\end{array}$ & - & - & - & $2(0.3 \%)$ \\
\hline & Escherichia & 522 & $522(100 \%)$ & $124(23.8 \%)$ & - & - & - & - & \\
\hline & Franconibacter & 10 & 10 (100\%) & - & - & - & - & - & \\
\hline & Klebsiella Clade A & 100 & $100(100 \%)$ & - & - & - & - & - & \\
\hline & Klebsiella Clade B & 310 & - & - & - & - & - & - & \\
\hline & Klebsiella Clade C & 189 & - & - & - & - & - & - & \\
\hline & Kluyvera & 9 & $9(100 \%)$ & $4(44.4 \%)$ & - & - & - & - & \\
\hline & Kosakonia & 24 & $24(100 \%)$ & - & - & $\begin{array}{c}15 \\
(62.5 \%)\end{array}$ & - & - & \\
\hline & Leclercia & 10 & 10 (100\%) & - & - & - & - & - & \\
\hline & Lelliottia & 13 & $13(100 \%)$ & $9(69.2 \%)$ & - & - & - & - & \\
\hline & Mangrovibacter & 2 & $2(100 \%)$ & $2(100 \%)$ & - & - & - & - & \\
\hline & Metakosakonia & 3 & $3(100 \%)$ & 1 (33.3\%) & - & - & - & - & \\
\hline & Phytobacter & 2 & $2(100 \%)$ & - & - & - & - & - & \\
\hline & Pluralibacter & 17 & 17 (100\%) & $2(11.8 \%)$ & - & $\begin{array}{c}14 \\
(82.4 \%)\end{array}$ & - & - & \\
\hline & Pseudescherichia & 2 & $2(100 \%)$ & $2(100 \%)$ & - & - & - & - & \\
\hline & Pseudocitrobacter & 1 & $1(100 \%)$ & $1(100 \%)$ & - & - & - & - & \\
\hline & Raoultella & 85 & - & - & - & - & - & - & \\
\hline & Salmonella & 112 & $112(100 \%)$ & - & - & - & - & - & \\
\hline & Shimwellia & 2 & - & - & - & - & - & - & \\
\hline & Siccibacter & 6 & $6(100 \%)$ & $2(0.33 \%)$ & - & - & - & - & \\
\hline & Superficieibacter & 2 & $2(100 \%)$ & - & - & - & - & - & \\
\hline & Trabulsiella & 8 & $8(100 \%)$ & - & - & - & - & - & \\
\hline & Yokenella & 5 & $5(100 \%)$ & - & - & - & - & - & \\
\hline
\end{tabular}


Table 1 Prevalence of the primary (flag-1) and additional (flag-2 to flag-5) loci among the Enterobacterales. The number and percentage of taxa at the family and genus level that incorporate each type of locus are indicated (Continued)

\begin{tabular}{|c|c|c|c|c|c|c|c|c|c|}
\hline Family & Genus & \# Strains & flag-1 & flag-2 & flag-3a & flag-3b & flag-4 & flag -5 & $\begin{array}{l}\text { \# Strains with } \\
3 \text { flag loci }\end{array}$ \\
\hline \multirow[t]{10}{*}{ Erwiniaceae } & & 287 & $\begin{array}{c}279 \\
(97.2 \%)\end{array}$ & $8(2.8 \%)$ & $\begin{array}{c}44 \\
(15.3 \%)\end{array}$ & $\begin{array}{c}12 \\
(4.2 \%)\end{array}$ & $\begin{array}{c}2 \\
(0.7 \%)\end{array}$ & $0(0 \%)$ & \\
\hline & Buchnera & 58 & $58(100 \%)$ & - & - & - & - & - & \\
\hline & Erwinia & 63 & 60 (95.2\%) & $2(3.2 \%)$ & 41 (65.1\%) & $1(1.6 \%)$ & - & - & \\
\hline & Izhakiella & 2 & $2(100 \%)$ & $2(100 \%)$ & - & - & - & - & \\
\hline & Mixta & 4 & $4(100 \%)$ & - & - & 4 & - & - & \\
\hline & Pantoea & 151 & $151(100 \%)$ & $2(1.3 \%)$ & $3(2 \%)$ & 7 (4.6\%) & - & - & \\
\hline & Phaseolibacter & 1 & - & - & - & - & - & - & \\
\hline & Rosenbergiella & 1 & $1(100 \%)$ & $1(100 \%)$ & - & - & - & - & \\
\hline & Tatumella & 5 & $3(60 \%)$ & $1(20 \%)$ & - & - & - & - & \\
\hline & Wigglesworthia & 2 & - & - & - & - & 2 & - & \\
\hline \multirow[t]{5}{*}{ Hafniaceae } & & 97 & 97 (100\%) & $28(28.9 \%)$ & $0(0 \%)$ & $4(4.1 \%)$ & $0(0 \%)$ & $0(0 \%)$ & 4 (4.1\%) \\
\hline & Edwardsiella & 50 & $50(100 \%)$ & - & - & - & - & - & \\
\hline & Enterobacillus & 1 & $1(100 \%)$ & - & - & - & - & - & \\
\hline & Hafnia & 42 & $42(100 \%)$ & $24(57.1 \%)$ & - & - & - & - & \\
\hline & Obesumbacterium & 4 & $4(100 \%)$ & $4(100 \%)$ & - & 4 & - & - & $4(100 \%)$ \\
\hline \multirow[t]{8}{*}{ Morganellaceae } & & 313 & $\begin{array}{c}311 \\
(99.4 \%)\end{array}$ & $0(0 \%)$ & $0(0 \%)$ & $1(0.3 \%)$ & $0(0 \%)$ & $0(0 \%)$ & \\
\hline & Arsenophonus & 2 & $2(100 \%)$ & - & - & - & - & - & \\
\hline & Moellerella & 2 & $2(100 \%)$ & - & - & - & - & - & \\
\hline & Morganella & 55 & $55(100 \%)$ & - & - & $1(1.8 \%)$ & - & - & \\
\hline & Photorhabdus & 31 & 31 (100\%) & - & - & - & - & - & \\
\hline & Proteus & 122 & $122(100 \%)$ & - & - & - & - & - & \\
\hline & Providencia & 58 & $56(96.6 \%)$ & - & - & - & - & - & \\
\hline & Xenorhabdus & 43 & 43 (100\%) & - & - & - & - & - & \\
\hline \multirow[t]{8}{*}{ Pectobacteriaceae } & & 244 & $\begin{array}{c}241 \\
(98.8 \%)\end{array}$ & $0(0 \%)$ & $0(0 \%)$ & $0(0 \%)$ & $\begin{array}{c}4 \\
(1.6 \%)\end{array}$ & $0(0 \%)$ & \\
\hline & Biostraticola & 1 & - & - & - & - & $\begin{array}{c}1 \\
(100 \%)\end{array}$ & - & \\
\hline & Brenneria & 9 & $9(100 \%)$ & - & - & - & - & - & \\
\hline & Dickeya & 55 & 55 (100\%) & - & - & - & - & - & \\
\hline & Lonsdalea & 35 & 35 (100\%) & - & - & - & - & - & \\
\hline & Pectobacterium & 140 & 138 (98.6\%) & - & - & - & - & - & \\
\hline & Samsonia & 1 & $1(100 \%)$ & - & - & - & - & - & \\
\hline & Sodalis & 3 & $3(100 \%)$ & - & - & - & $\begin{array}{c}3 \\
(100 \%)\end{array}$ & - & \\
\hline \multirow[t]{2}{*}{ Thorselliaceae } & & 1 & $1(100 \%)$ & $0(0 \%)$ & $0(0 \%)$ & $0(0 \%)$ & $0(0 \%)$ & $0(0 \%)$ & \\
\hline & Thorsellia & 1 & $1(100 \%)$ & - & - & - & - & - & \\
\hline \multirow[t]{6}{*}{ Yersiniaceae } & & 605 & $\begin{array}{c}593 \\
(97.9 \%)\end{array}$ & $\begin{array}{c}225 \\
(37.2 \%)\end{array}$ & $0(0 \%)$ & $\begin{array}{c}44 \\
(7.3 \%)\end{array}$ & $0(0 \%)$ & $0(0 \%)$ & $8(1.3 \%)$ \\
\hline & Chania & 2 & $2(100 \%)$ & $1(50 \%)$ & - & - & - & - & \\
\hline & Ewingella & 2 & $2(100 \%)$ & - & - & - & - & - & \\
\hline & Gibbsiella & 4 & - & - & - & - & - & - & \\
\hline & Nissabacter & 1 & $1(100 \%)$ & - & - & - & - & - & \\
\hline & Rahnella & 18 & 17 (94.4\%) & - & - & $4(22.2 \%)$ & - & - & \\
\hline
\end{tabular}


Table 1 Prevalence of the primary (flag-1) and additional (flag-2 to flag-5) loci among the Enterobacterales. The number and percentage of taxa at the family and genus level that incorporate each type of locus are indicated (Continued)

\begin{tabular}{|c|c|c|c|c|c|c|c|c|c|c|}
\hline Family & Genus & \# Strains & flag-1 & flag-2 & flag-3a & flag-3b & flag-4 & flag-5 & $\begin{array}{l}\text { \# Strains with } \\
3 \text { flag loci }\end{array}$ & \\
\hline & Rouxiella & 3 & - & $1(33.3 \%)$ & - & - & - & - & & \\
\hline & Serratia Clade A & 159 & 157 (98.7\%) & - & - & - & - & - & & \\
\hline & Serratia Clade B & 12 & $12(100 \%)$ & - & - & $1(8.3 \%)$ & - & - & & \\
\hline & Serratia Clade C & 8 & 8 (100\%) & - & - & - & - & - & & \\
\hline & Serratia Clade D & 1 & - & - & - & - & - & - & & \\
\hline & Serratia Clade E & 1 & - & - & - & - & - & - & & \\
\hline & Yersinia & 394 & 394 (100\%) & 223 (56.6\%) & - & $39(10 \%)$ & - & - & $8(2.0 \%)$ & \\
\hline \multirow[t]{2}{*}{$\begin{array}{l}\text { Family } \\
\text { Unassigned }\end{array}$} & & 8 & $0(0 \%)$ & 8 (100\%) & $0(0 \%)$ & $0(0 \%)$ & $0(0 \%)$ & $\begin{array}{c}8 \\
(100 \%)\end{array}$ & & \\
\hline & Plesiomonas & 8 & - & $8(100 \%)$ & - & - & - & $8(100 \%)$ & & \\
\hline OVERALL & & 4028 & & $\begin{array}{c}3400 \\
(84.4 \%)\end{array}$ & $\begin{array}{c}593 \\
(14.7 \%)\end{array}$ & $\begin{array}{c}154 \\
(3.8 \%)\end{array}$ & $\begin{array}{c}95 \\
(2.4 \%)\end{array}$ & $\begin{array}{c}6 \\
(0.1 \%)\end{array}$ & $\begin{array}{l}8 \\
(0.2 \%)\end{array}$ & $\begin{array}{c}19 \\
(0.5 \%)\end{array}$ \\
\hline
\end{tabular}

loci (Table 1). Eighteen of these harbour a flag-1, flag-2 and flag-3 locus and include members of the families Enterobacteriaceae (one strain of Buttiauxella warmboldiae, one strain of Enterobacter and four strains of Citrobacter Clade C), Hafniaceae (four strains of Obesumbacterium proteus) and Yersiniaceae (eight Yersinia spp.) (Table 1; Fig. 2). One strain, Enterobacter ludwigii OLC-1682, lacks a flag-2 locus, but instead incorporates one flag-1 locus and two flag-3 loci (discussed in further detail below).

Comparison of the flag-1 through to flag-5 loci revealed that, while there is some synteny in gene blocks between the distinct flag loci (flag-1 through to flag-5), there is also evidence of extensive rearrangements and inversion of gene blocks (Fig. 3). Furthermore, some of the flag loci show evidence of gene deletion events, while in others additional, non-conserved genes have been integrated within the loci (Fig. 3). Average amino acid identity (AAI) values across the 25 SCOs shared among all flag loci further supports the separation of the enterobacterial flag loci into five distinct types with intra-clade AAI values ranging between $57.79 \%$ (flag-4 loci) and 99.06\% (flag-5 loci), while the inter-clade values are only between 32.80 and 52.23\% (Additional file 1: Table S2). These data suggest distinct evolutionary histories for the flag- 1 to flag-5 loci. As such, a more in depth analysis of the flag-3, -4 and -5 loci was undertaken.

\section{The flag-3 peritrichous flagellar loci can be further} divided into two subtypes, flag-3a and flag-3b on the basis of sequence synteny and conservation

The flag-3 loci cluster with the peritrichous primary flagellar (flag-1) loci in the SCO phylogeny (Fig. 1) and they share $52.23 \%$ average amino acid identity across the twenty-five conserved SCO proteins encoded on the loci.
The former loci incorporate genes coding for $46 / 47$ proteins involved in flagellar biosynthesis, regulation and maintenance and chemotaxis in the primary flagellar locus. One exception is the absence of orthologues of the gene coding for FliZ in the flag-3 loci. In the flag-1 loci FliZ is an activator for the expression of class $2 /$ middle genes involved in the synthesis in the flagellar hook and basal body [17]. A distinct regulatory system for class 2 gene expression may occur in the flag-3 system.

Phylogenetic evaluation of the flag-3 loci, on the basis of the concatenated alignment of 45 SCOs (excluding FliC which is present in multiple copies in some strains) conserved amongst them, showed that they fall into two distinct clades (Fig. 4), with the loci of the upper clade (flag-3a) comprising 154 (61.6\% of total flag-3 loci) and the lower clade (flag-3b) comprising 95 taxa. The flag-3a loci are restricted to members of three genera in two enterobacterial families (the Enterobacteriaceae and Erwiniaceae), namely Enterobacter (111/608 of the studied strains), Erwinia (41/61 of the studied taxa) and Pantoea (3/151 studied taxa) (Table 1). By contrast, the flag-3b locus is represented across a much broader taxonomic spectrum, including the Enterobacteriaceae (one Buttiauxella, four Citrobacter Clade D, fifteen Kosakonia and fourteen Pluralibacter strains), Erwiniaceae (one Erwinia, three Mixta and seven Pantoea strains), Hafniaceae (four Obesumbacterium proteus strains), Morganellaceae (Morganella sp. nov. $2 \mathrm{H} 1 \mathrm{r}$ ) and Yersiniaceae (four Rahnella, one Serratia Clade B and 39 Yersinia strains) (Table 1). The presence of these flag-3 loci appears to be mutually exclusive, with no taxon containing both flag-3a and flag-3b loci. There is, however, one strain, Enterobacter ludwigii OLC-1682 which incorporates two flag-3a loci. These cluster together with the other Enterobacter sp. flag-3a loci, but in distinct sub- 


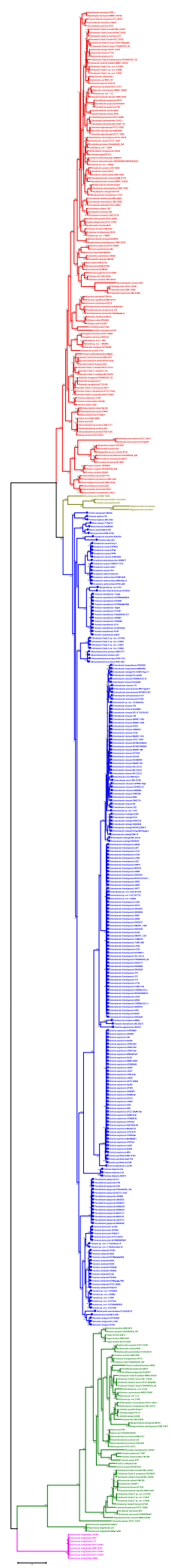

Fig. 1 Phylogenetic differentiation of five distinct flagellar (flag) loci among the Enterobacterales. A ML phylogeny was constructed on the basis of 25 SCOs conserved among all flag loci. The trimmed alignment was comprised of 4523 amino acid sites and the best-fit evolutionary model $\mathrm{LG}+\mathrm{F}+\mathrm{I}+\mathrm{G} 4$ was selected for phylogeny construction. Bootstrap values $(n=1000)$ are shown. The flag-1 (red), flag-2 (green), flag-3 (blue), flag-4 (olive) and flag-5 (pink) clades are indicated by the distinct branch colours

clades, with flag-3a-1 clustering with those of E. bugandensis and flag-3a-2 clustering with the other E. ludwigii loci. They furthermore share $84.42 \%$ AAI values (entire Enterobacter clade $=84.12 \%$ AAI), suggesting that $E$. ludwigii OLC-1682 derived these loci through distinct evolutionary events.

The flag-3a loci are on average $46.3 \mathrm{~kb}$ in size, have an average $\mathrm{G}+\mathrm{C}$ content of $55.0 \%$ and code for 51 distinct proteins, while the flag-3b loci are $\sim 44.3 \mathrm{~kb}$ in size, have an average $\mathrm{G}+\mathrm{C}$ content of $50.5 \%$ and code for 48 proteins (Additional file 1: Table S3). The proteins encoded on the two subtypes, flag-3a and flag-3b, share distinct sequence conservation, with intraclade AAI values of $75.54 \%$ (flag-3a) and $69.09 \%$ (flag-3b), respectively, while the inter-clade AAI value is $59.01 \%$ (on the basis of 25 conserved SCOs) (Additional file 1: Table S2). Furthermore, the flag-3a and flag-3b loci have distinct gene syntenies. The flag-3a loci all comprise of three gene blocks occurring in the order block 1: flhEAB-cheZYBR-fliEFGHIJKLMNOPQR, block 2: fliCD $S T$ and block 3: flgNMABCDEFGHIJKL-fliA-flhDC-motABche $A W$. In most of the flag-3b loci block 3 precedes block 2 and block 1 is situated at the $3^{\prime}$ end of the locus. Two notable exceptions are the flag-3b loci of Buttiauxella warmboldiae CCUG 35512 and Morganella sp. nov 2 H1r. In both taxa, gene block 3 occurs at the $5^{\prime}$ end of the locus, but block 2 is integrated within gene block 1 , with part of the latter gene block occurring in reverse complement (Fig. 5). The co-localisation of the genes in their gene blocks, regardless of the locus subtype, suggests these loci may have been built by the step-wise incorporation of the individual gene blocks, which may have been derived from distinct ancestral loci. This is supported by the distinct $\mathrm{G}+$ $\mathrm{C}$ contents of the gene blocks. The $\mathrm{G}+\mathrm{C}$ content of gene block 1 is on average 4.45 and $2.23 \%$ higher than those of block 2 and block 3 of the flag-3 loci (both flag-3a and flag$3 b)$, respectively.

Similar to what is observed in both flag-1 and flag-2 loci $[12,15]$, the flag-3a and flag-3b loci are characterized by the presence of a non-conserved genomic island adjacent to the $\mathrm{fliC}$ gene (Fig. 5). This island occurs in 150/155 of the flag-3a loci and 78/95 of the flag-3b loci, has an average size of $3.9 \mathrm{~kb}$ (range: $0.5-8.9 \mathrm{~kb}$ ) and codes for between one and eight distinct cargo proteins 


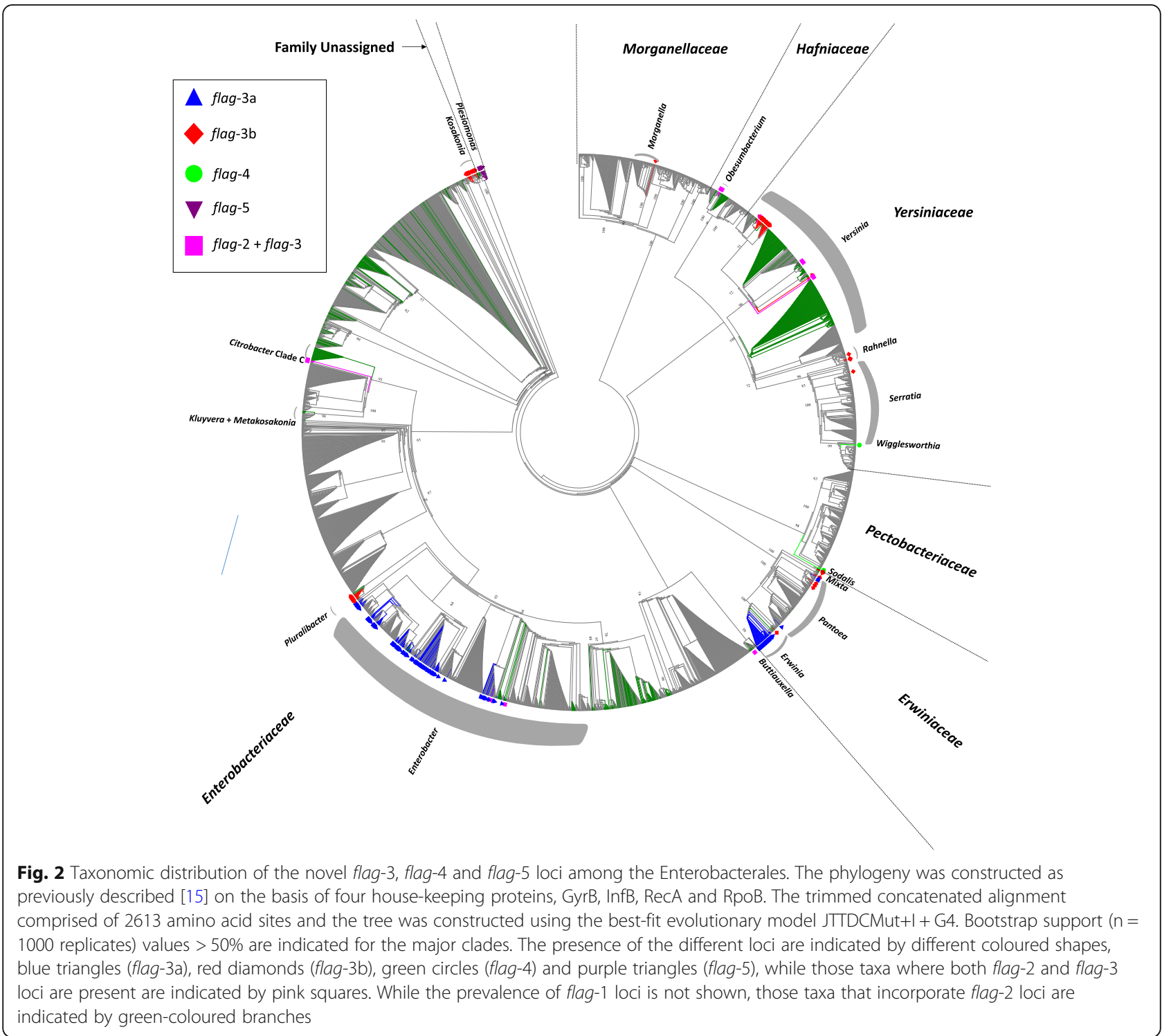

(Additional file 1: Table S3). A total of twenty-seven distinct proteins are encoded on this island, with nine of these unique to the flag-3a loci and seventeen unique to the flag-3b loci (Additional file 1: Table S4). One island feature is shared between the islands of 15 flag-3a and 57 flag-3b loci and codes for a methyl-accepting chemotaxis protein (Mcp1; 45.26\% average amino acid identity; COG0840). A second methyl-accepting chemotaxis protein (Mcp2; 86.22\% average amino acid identity; PRK15048) is found in the flag-3a islands of Enterobacter and Pantoea spp. A key feature among the Enterobacterales is the presence of genes in the flagellar locus coding for proteins with roles in glycosylation and posttranslational modification of the main flagellar structural protein, flagellin. Flagellin glycosylation has been linked to a number of functions, including flagellar synthesis and stabilization, biofilm formation, surface recognition and adherence, virulence and host immune evasion [18, 19]. Previous studies that showed $17.4 \%(307 / 1761)$ and $57.6 \%(341 / 592)$ of the flag-1 and flag-2 loci incorporated flagellin glycosylation machinery, respectively [12, 15]. Among the flag-3 loci, only the flag-3a loci of 41 (26.45\% of the flag-3a loci; $16.4 \%$ of total flag-3 loci) Erwinia spp. incorporate three genes coding for enzymes involved in flagellin modification adjacent to the $\mathrm{fliC}$ gene (Fig. 5). One gene codes for a 1127 amino acid (96.45\% AAI) N-acetyl glucosamine glycosyltransferase (Spy), which catalyses the post-translational addition of $\mathrm{O}$-linked beta- $\mathrm{N}$-acetylglucosamine to serine/threonine residues in the target protein (Additional file 1: Table S4) [20]. The flagellin glycan chains are frequently further decorated by formyl, methyl, acetyl and amino 


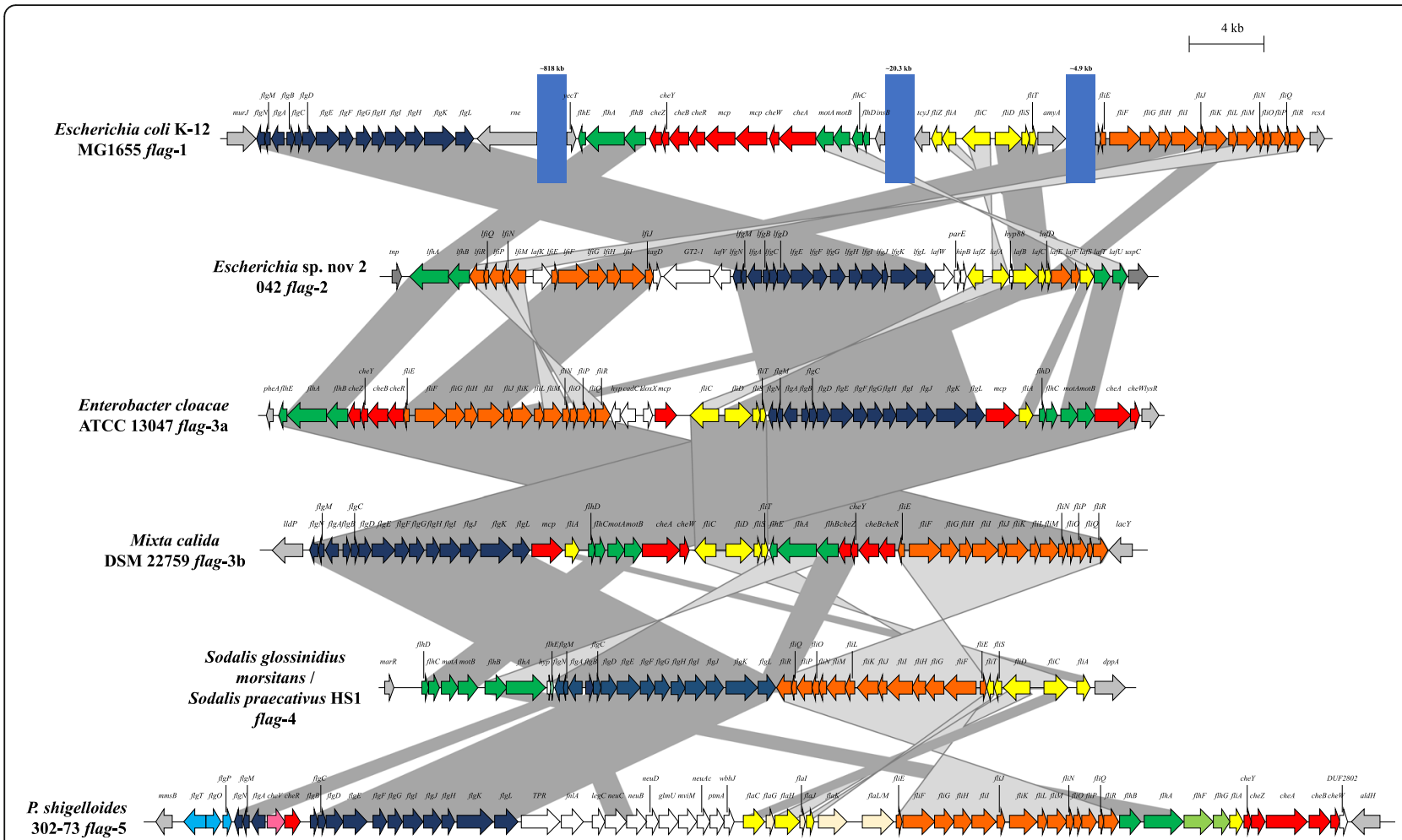

Fig. 3 Schematic comparison of the flag-1 to flag-5 loci. The flag loci genes are coloured according to orthology to conserved genes in the flag-1 locus, with flanking genes coloured in grey, and non-conserved cargo genes in white. The blue blocks indicate chromosomal gaps between the flag-1 locus regions. The scale bar (4 kilobases) indicates the relative sizes of the loci

groups [12]. Adjacent to the spy gene in the Erwinia flag-3a are genes coding for an aminotransferase (WecE; 98.01\% AAI; COG0399) and O-acetyltransferase (WbbJ; 93.65\% AAI; COG0110) suggesting that the Erwinia flag-3b flagellin glycan is both acetylated and aminated, while the Spy protein also incorporated a methyltransferase domain (pfam13649) (Additional file 1: Table S4).

A key feature of the non-conserved island adjacent to the $\mathrm{fliC}$ gene among the flag-3a loci is the universal presence of a gene coding for a transcriptional regulator, CadC1 (COG3710) (Additional file 1: Table S4). Given its position in the locus, where fliZ occurs in the flag-1 locus, it is plausible that this transcriptional regulator may serve a similar role in the flag-3a loci, but this will need to be validated experimentally. No gene with this purported function is present in the flag-3b loci and hence how the class 2 gene expression would be regulated in the latter loci remains unclear.

\section{The flag-4 locus is predominant among insect} endosymbionts and codes for a predicted peritrichous flagellum

The flag-4 loci cluster with the flag-1 and flag-3 loci in the flagellar SCO phylogeny and share 49.69 and 46.73\%
AAI values with the former and latter loci, respectively (Fig. 1; Additional file 1: Table S2). BlastP analysis of the flag-4 protein complement revealed the closest matches are proteins encoded on the flag-1 and flag-3 loci, suggesting that the flag-4 locus likewise codes for a peritrichous flagellar system. Of all the flag systems, the flag-4 loci show the greatest versatility, ranging in size between 31.6 and $45.1 \mathrm{~kb}$ and $\mathrm{G}+\mathrm{C}$ content between 22.5 and $56.1 \%$ with an intra-clade AAI value of $57.79 \%$ (across 25 conserved SCOs) (Additional file 1: Tables S2 and S3). Furthermore, while the flag-4 loci share extensive synteny, the flag-4 loci show evidence of frequent deletions and gene disruption through transposon integration (Fig. 6). As such, the flag-4 locus of the tsetse fly endosymbiont Sodalis glossinidius 'morsitans B4' lacks the cheZYBR-cheAW and fliZ genes and incorporates a pseudogene of $f l h E$, while that of S. pierantonius SOPE (Sitophilus oryzae endosymbiont) lacks the genes cheZYBR-cheAW, flhE, flgB, fliZST and incorporates pseudogene copies of $f l g C$, fliQ, fliE, fliC and fliD. Similarly, while their genomes incorporate flag-1 loci, they are likewise heavily degraded (Fig. 6). The eroded flag-1 and flag-4 loci in these Sodalis strains are typical of the observed degenerative genome evolution as these bacteria adapted to a symbiotic lifestyle and indeed they 

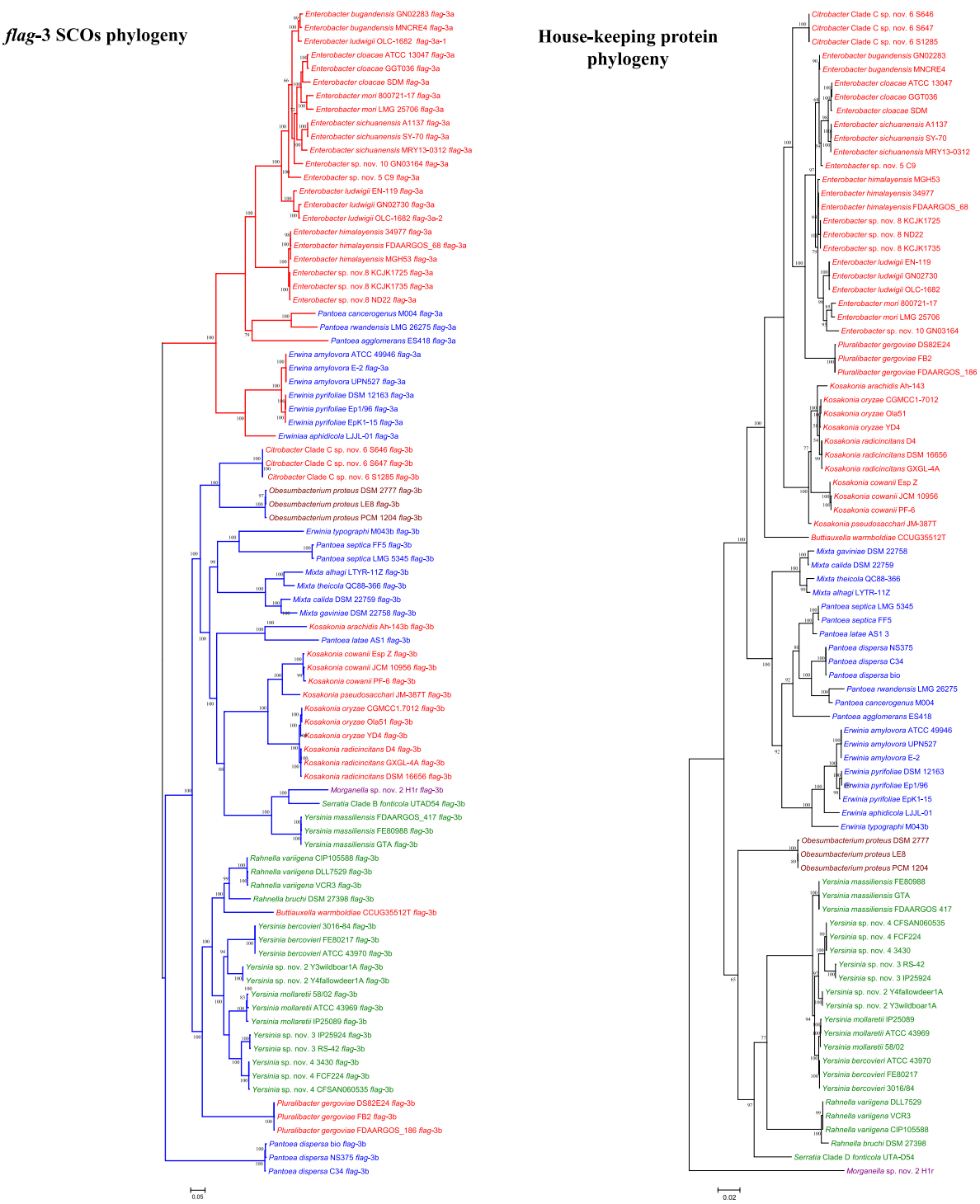

Fig. 4 Phylogeny of flag-3 loci compared to house-keeping protein tree. ML phylogenies were constructed on the basis of the concatenated alignments of $45 \mathrm{SCO}$ conserved among the flag-3 loci (left) and the house-keeping proteins GyrB, InfB, RecA and RpoB (right). The final alignments comprised of 10,357 and 3086 amino acids, respectively. The best-fit evolutionary model LG +F + I + G4 was used for construction of both trees and bootstrap support ( $n=1000$ replicates) values are shown. The taxa names are coloured according to their family designation, while the flag-3a and flag-3b loci branches are coloured in red and blue, respectively in the flag-3 SCO phylogeny

have been described as non-motile [21, 22]. The gene complement of the flag- 4 locus of Sodalis praecaptivus HS1 reflects that observed in S. glossinidius 'morsitans B4', and it also harbours a complete flag-1 locus (encodes $46 / 47$ of the primary flagellar proteins, with the exception of FliZ). This latter strain, differs from the insect symbiont Sodalis spp. in that it was isolated from a human wound, can persist in free-living form and has been observed to be capable of swarming motility [23, 24], although whether the flag-1 or flag-4 locus encoded flagellar system is responsible for this capacity remains to be elucidated.
The two tsetse fly endosymbiotic Wigglesworthia glossinidia strains included in this study lack flag-1 loci but both incorporate flag-4 loci. The loci of these two strains are the smallest among the flag-4 loci (Additional file 1: Table S3) and include only 33/47 genes coding for orthologues of the primary flagellar (flag-1) locus proteins, lacking the chemotaxis genes cheZYBR-cheAW, as well as the flagellar biosynthetic and regulatory genes flhE, flgM and fliATZ (Fig. 6). As such, the resultant flagellar system would be expected to be non-functional as is the case in the endosymbiotic Sodalis spp. However, gene expression analysis of the $\mathrm{fliC}$ (flagellin) 


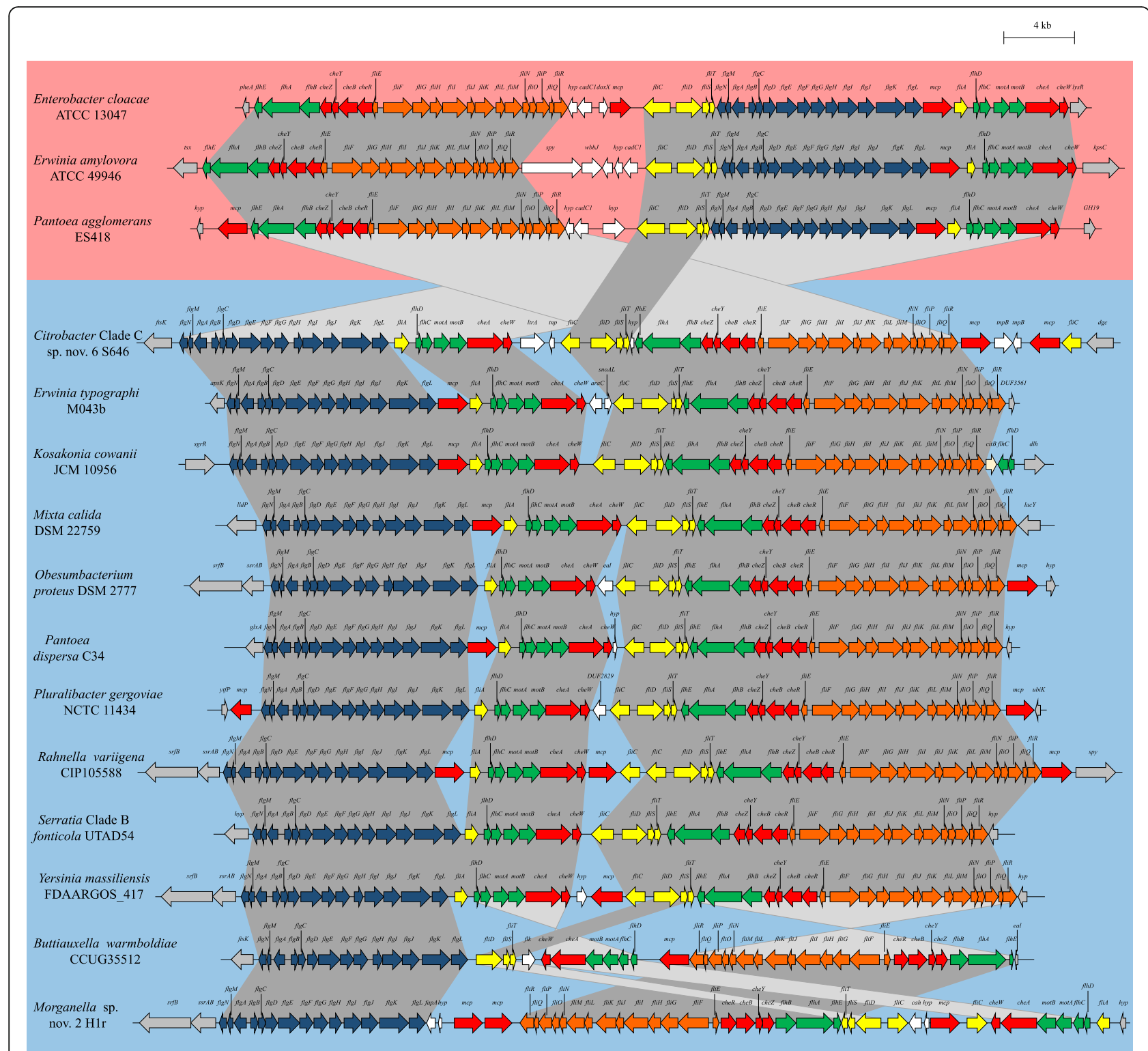

Fig. 5 Schematic comparison of the flag-3 loci. The flag loci genes are coloured according to orthology to conserved genes in the flag-1 locus, with flanking genes coloured in grey, and non-conserved cargo genes in white. The flag-3a loci are background shaded pink, while the flag-3b loci are shaded light blue. The scale bar (4 kilobases) indicates the relative sizes of the loci

and $\operatorname{mot} A$ (motor protein $\mathrm{A}$ ) genes and immunohistochemistry analysis with flagellins-specific antibodies showed the expression of both and production of flagellin in intrauterine larvae and the milk gland cells of tsetse flies, suggesting an important role for the flag-4 flagellum in Wigglesworthia vertical transmission from host mother to progeny [25]. Similarly, Biostraticola tofi DSM 19580, isolated from biofilm on a tufa limestone deposit has been shown to synthesise flagella and be capable of swimming motility [26]. As the genome of this bacterium solely incorporates a flag-4 locus, a role for the flagellar system it encodes in motility can be suggested for the Biostraticola and Wigglesworthia.

\section{The flag-5 locus is unique to Plesiomonas shigelloides among the Enterobacterales and codes for a polar flagellum}

Plesiomonas shigelloides lack flag-1 loci, but previous studies have identified a lateral flag-2 locus in this species $[15,16]$. Furthermore, they incorporate the distinct flag-5 locus that forms a separate clade in the SCO phylogeny (Fig. 1). The proteins encoded by this locus share 


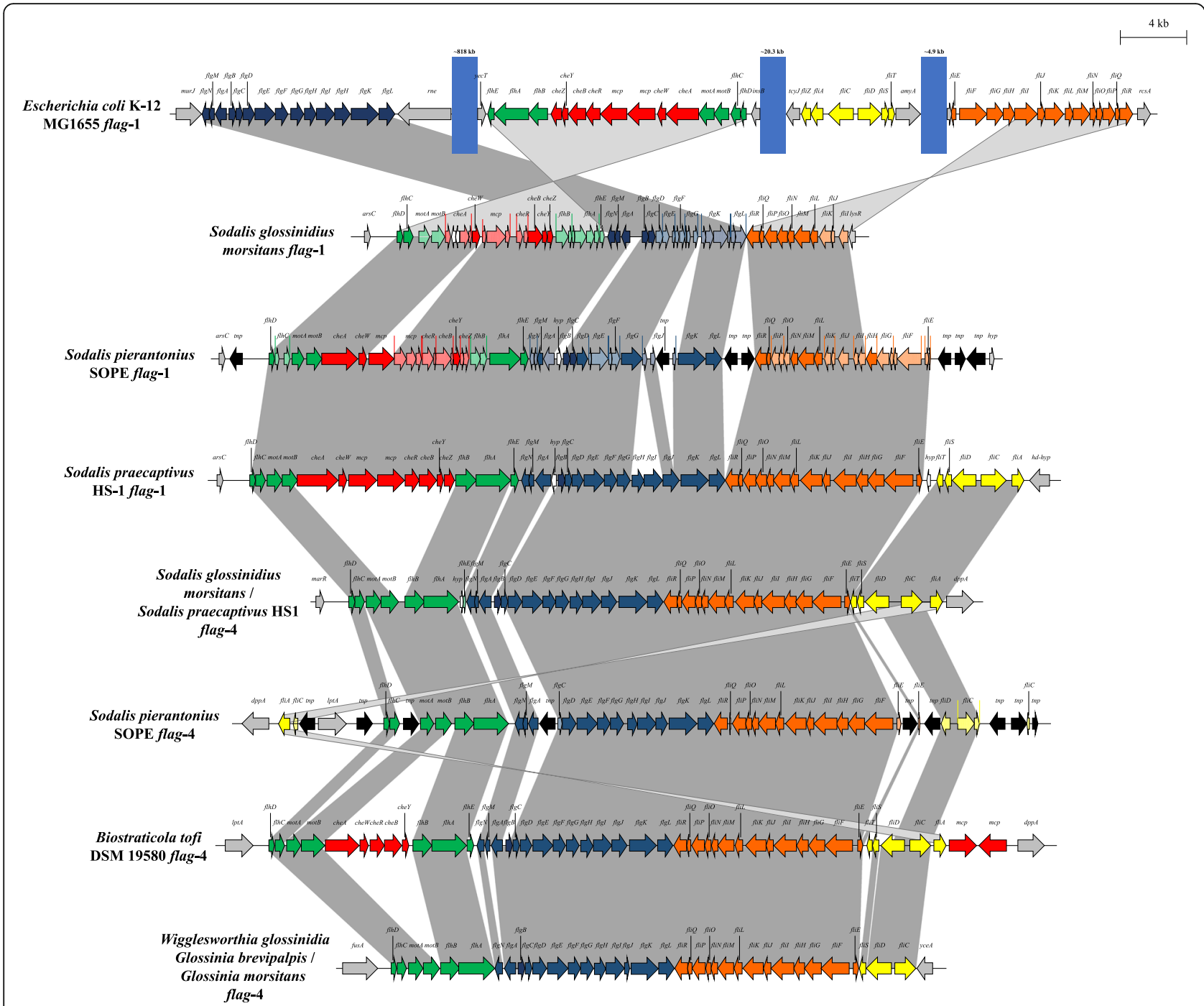

Fig. 6 Schematic comparison of the flag-4 loci and Sodalis flag-1 loci. The flag loci genes are coloured according to orthology to conserved genes in the flag-1 locus, with flanking genes coloured in grey, non-conserved cargo genes in white and transposase genes in black. Predicted pseudogenes are shown as hashed arrows. The blue blocks indicate chromosomal gaps between the flag-1 locus regions. The scale bar (4 kilobases) indicates the relative sizes of the loci

limited sequence identity with those encoded on the other flag loci, with AAI values ranging between $32.80 \%$ (flag-4) and $34.92 \%$ (flag-1) across the 25 SCOs conserved among the loci (Additional file 1: Table S2). Instead, they share $54.95 \%$ AAI across 52 proteins with the polar flagellar loci of Vibrio parahaemolyticus BB22O (AF069392.3 and U12817.2) [16]. Furthermore, the flag5 locus shows extensive synteny with the polar flagellar locus of the latter strain (Fig. 7). While the V. parahaemolyticus polar flagellar system is encoded by two loci, which are separated by $\sim 1.45$ megabases on the chromosome, the genes for polar flagellar synthesis in $P$. shigelloides are harboured in a single locus, which ranges between 57.9 and $62.0 \mathrm{~kb}$ in size and codes for 57-61 distinct proteins among the eight $P$. shigelloides incorporated in this study. These loci also represent the largest among the flag-1 to flag-5 loci.

Vibrio parahaemolyticus encodes two flagellar systems which facilitate movement under distinct conditions, with the lateral flagella (multiple) allowing swarming motility across surfaces, while the single polar flagellum enables swimming in liquid environments [7, 27]. The former are powered by the proton motive force, as observed for the peritrichous (flag-1) flagella of the Enterobacterales, while the latter derive their energy through the sodium membrane potential [7, 27]. A previous study has shown that $P$. shigelloides is likewise capable of both swarming and swimming motility, with the 


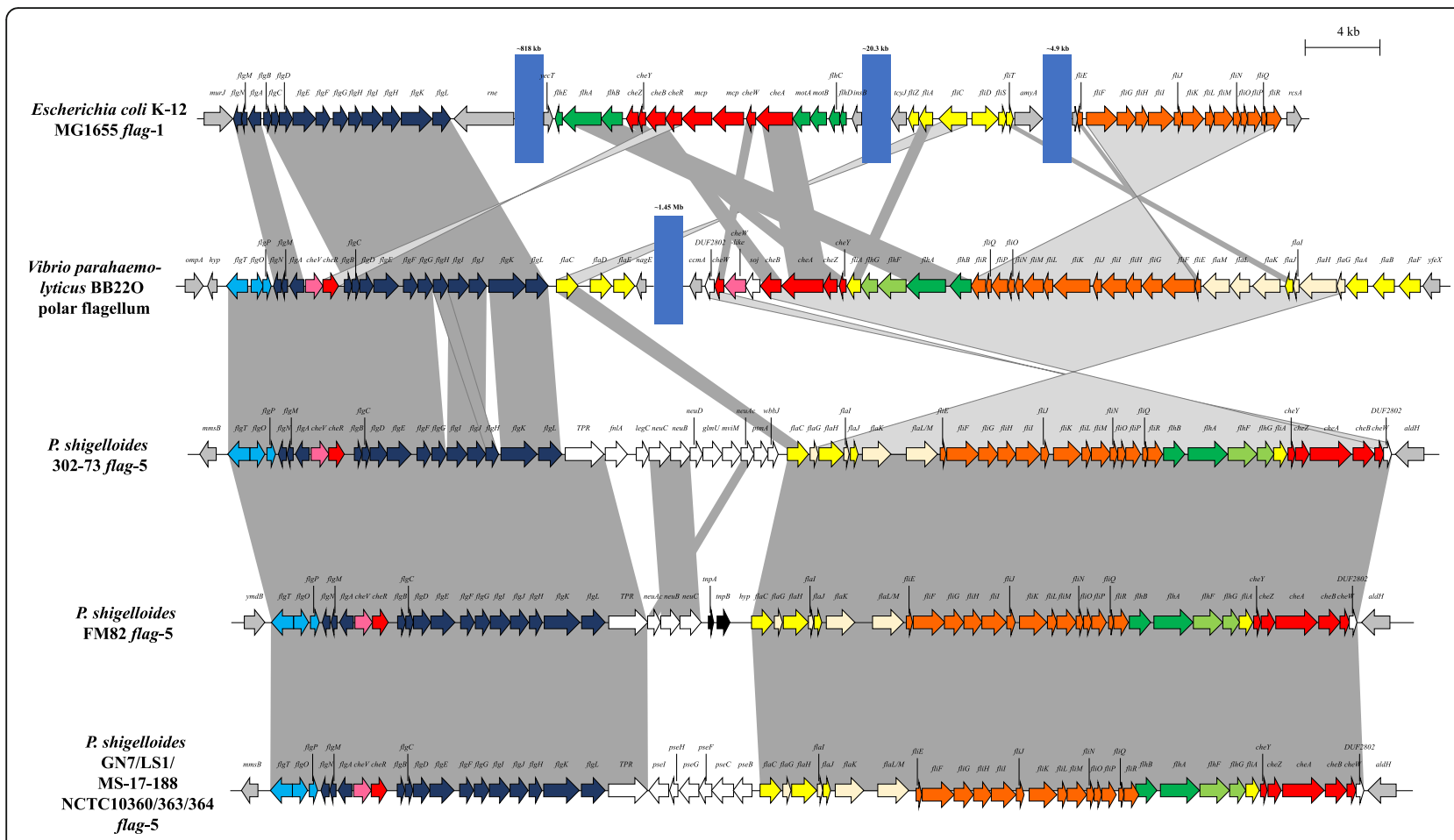

Fig. 7 Schematic comparison of the flag-5 loci and V. parahaemolyticus BB22O polar flagellar locus. The flag loci genes are coloured according to orthology to conserved genes in the flag-1 locus, with flanking genes coloured in grey, non-conserved cargo genes in white and transposase genes in black. The blue blocks indicate chromosomal gaps between the flag-1 locus regions. The scale bar (4 kilobases) indicates the relative sizes of the loci

flag-2 and flag-5 linked to the former and latter form of motility, respectively [16].

Comparison of the protein complement with those of the other Enterobacterales flag loci identified twentynine proteins that are unique to the flag- 5 loci, although seven of these share orthology with proteins encoded by the $V$. parahaemolyticus locus. Three of these orthologues, $\mathrm{FlgO}, \mathrm{FlgP}$ and $\mathrm{FlgT}$ (30.04-48.83\% AAI with $V$. parahaemolyticus BB22O AGB09241.1-244.1) are predicted to form part of the $\mathrm{H}$ ring, an additional basal body ring that is associated with the outer membrane. The H-ring is specific to Vibrio spp. and facilitates outer membrane penetration and external assembly of the sheathed polar flagella [27, 28]. The Vibrio polar flagellar locus lacks the genes $f l h C$ and $f l h D$ coding for the master transcriptional regulators in the enterobacterial peritrichous flagella. Instead the Vibrio polar flagellar loci incorporate three genes, flaK, flaL and flaM which are purported to fulfil this function. FlaK is a $\sigma^{54}$-dependent transcription factor of FlaL and FlaM. The histidine kinase-like FlaL protein then phosphorylates FlaM which activates the transcription of the flagellar middle class genes [10]. The Plesiomonas FlaK orthologues share $58.15 \%$ AAI with that of $V$. parahaemolyticus $\mathrm{BB} 22 \mathrm{O}$ (AGB10537.1). However, instead of two distinct FlaL and FlaM proteins, the flag-5 locus of $P$. shigelloides encodes a single 558 aa protein, FlaLM, with a histidine kinase domain (cd00082; Bitscore: 41; E-value 8.47e-5) and a $\sigma^{54}$-activator domain (pfam00158; Bitscore: 295; E-value: $3 e-98$ ) at the $\mathrm{C}$ and $\mathrm{N}$ terminal, respectively. The FlaLM protein appears to be the product of a gene fusion between flaL and flaM, with the first 120 aa sharing $56.08 \%$ AAI with the $V$. parahaemolyticus FlaL protein (AGB10535.1; aa 1-184) and aa 200-558 sharing $59.24 \%$ AAI with the $V$. parahaemolyticus FlaM protein (AGB10536.1; aa 121-468). Both the P. shigelloides flag5 and $V$. parahaemolyticus $\mathrm{BB} 22 \mathrm{O}$ polar flagellar loci lack orthologues of the chaperone protein FliT. However, both incorporate a gene, flaI, adjacent to the fliS orthologues, which encodes a protein that is similar in size to FliT and hence may perform the chaperone function [7]. Furthermore, both encode a chemotaxis related protein, $\mathrm{CheV}$, a CheY-CheW hybrid protein which is absent in the Enterobacterales flag loci [7].

A key difference between the $P$. shigelloides flag-5 loci and the $V$. parahaemolyticus $\mathrm{BB} 22 \mathrm{O}$ polar flagellar locus is the presence of six distinct orthologues of the flagellin protein, FlaA-F in the latter strain. The P. shigelloides flag-5 loci include one orthologue, which shows highest sequence identity with the $\mathrm{FlaC}$ protein in $V$. 
parahaemolyticus BB22O (AGB09262.1; 51.85\% AAI). By contrast, the $P$. shigelloides flag-5 loci uniquely incorporate between four and eleven genes adjacent to the flaC gene (Fig. 7; Additional file 1: Table S5). In P. shigelloides 302-73, these genes have been shown to code for proteins involved in the synthesis of the legionaminic acid, which posttranslationally glycosylates the flagellin protein [16]. Mutagenesis shows this glycan to be essential for biosynthesis of the flagellum in this strain [16]. Distinct flagellin glycosylation proteins are encoded in the other flag-5 loci. P. shigelloides FM82, incorporates three genes coding for the proteins NeuAc (acylneuraminate cytidylyltransferase), NeuB (N-acetylneuraminate synthase) and NeuC (UDP-N-acetyl-D-glucosamine 2epimerase), involved in the synthesis of neuraminic acid, the precursor for legionaminic acid (Additional file 1: Table S5). These proteins share only $31.16 \%$ AAI with its orthologues in the P. shigelloides 302-73 flag-5 locus, suggesting that a distinct flagellin glycan is present in $P$. shigelloides FM82. The six other strains incorporated in this study code for orthologues of PseB (UDP-N-acetylglucosamine 4,6dehydratase), PseC (UDP-4-amino-4,6-dideoxy-N-acetylbeta-L-altrosamine transaminase), PseF (pseudaminic acid cytidylyltransferase), PseG (UDP-2,4-diacetamido-2,4,6-trideoxy-beta-L-altropyranose hydrolase), PseH (UDP-4amino-4,6-dideoxy-N-acetyl-beta-L-altrosamine Nacetyltransferase) and PseI (UDP-4-amino-4,6-dideoxy-Nacetyl-beta-L-altrosamine $\mathrm{N}$-acetyltransferase) (Additional file 1: Table S5), the six enzymes which constitute the pathway for the synthesis of pseudaminic acid that forms part of the flagellin glycan in a number of both Gram-negative (Campylobacter and Helicobacter spp.) and Gram-positive (Geobacillus spp.) taxa [29, 30]. These proteins share $97.41 \%$ AAI among the six P. shigelloides flag-5 loci that code for them, with the exception of PseI, where the orthologue in P. shigelloides NCTC 10360 shares only 29\% AAI with those in the other five strains (97.42\% AAI). This highlights that, while flagellin glycosylation appears to be a universal feature of the $P$. shigelloides flag-5 system, it is highly versatile both in the type of sugar and decoration of the glycan.

\section{Discussion}

Flagellar motility, a common feature among most members of the Enterobacterales, was long considered to derive from a single chromosomally encoded peritrichous flagellar system (flag-1). However, a second, distinct lateral flagellar system (flag-2) was recently identified and shown to be fairly common across most family lineages within the order $[14,15]$. Here we have identified three additional distinct flagellar loci, flag-3 to flag-5, with discrete taxonomic distributions. These loci range in size between 31.6 and $62.0 \mathrm{~kb}$ and code for between 36 and 61 proteins. Research on the flag-1 system in E. coli showed that the assembly of the thirty proteins (including $\sim 20,000$ copies of the filament protein $\mathrm{FliC}$ ) that make up the primary flagellum structure is an energetically expensive process, contributing $\sim 2 \%$ of the total energy burden of the cell [3]. Furthermore, rotation of the flagellum consumes ion motive forces which could power other cellular processes [13, 31]. As such, flagellar synthesis and motility is a tightly regulated at multiple levels [13, 31]. Furthermore, proteins involved in flagellar biosynthesis and function contribute on average $1.08 \%$ to the overall proteome (number of distinct flagellar proteins/total number of distinct proteins encoded on genome) and this already substantial proportion increases on average to $2.06 \%$ and up to $3.20 \%$ of the proteome when two or three flag loci are present, respectively. Thus the maintenance and functioning of two and sometimes three distinct flagellar systems would substantively increase the metabolic burden on the cell and raises questions on their evolution, functioning and biological roles among members of the Enterobacterales.

Previous studies have postulated that the primary (flag-1) flagellar system evolved from a single precursor or a few genes, which have subsequently undergone gene duplications and gene fusions to give rise to the current complement of genes required for flagellar synthesis. This is based on the extensive sequence conservation observed among twenty-four core flag-1 proteins in 41 motile taxa across eleven bacterial phyla [6, 32]. Similarly, the minimal gene set purported to have formed the primary flagellar system may have been duplicated elsewhere in the genome of many of the Enterobacterales. This may have given rise to the distinct clades represented by 1 ) the flag-1, -3 and -4 loci, 2) the flag-2 loci and 3) the flag-5 loci, which may subsequently have diversified through the recruitment of novel genes. For example, the flag-2 loci uniquely incorporate genes coding for the regulatory proteins LafK and LafZ and the hookassociated protein LafW, while the $f \lg O, f \lg P$ and $f l g T$ genes coding for the $\mathrm{H}$-ring and the regulatory genes flaK and flaLM are unique to flag-5 loci. This evolutionary hypothesis seems plausible for the flag-2 locus, which was previously shown to be prevalent among most enterobacterial lineages prior to extensive loss through deletion events [15]. By contrast, the polar flag-5 locus is unique among the Enterobacterales to strains of $P$. shigelloides, suggesting it may have been acquired through horizontal gene transfer, possibly from a member of the Vibrionaceae. The flag-1, -3 and -4 loci show a more convoluted relationship. These three loci also show extensive sequence conservation and synteny, with three gene blocks, flgNMABCDEFGHIJKL, fliEFGHIJKLM$N O P Q R$ and fliCDST (Fig. 3) universally present in each, while the flag-1 locus flhEAB-cheZYBR-cheAW-motAB$f l h D C$ gene block is interspersed between these 
conserved blocks in the flag-3 and -4 loci. It is thus likely that the latter loci have been derived through duplications of the gene blocks and integration into an available genomic site, albeit in a different order from the flag-1 system. Notable is that the flag-4 loci in the three Sodalis spp. incorporated in this study display greater synteny with the flag-1 loci in these taxa, rather than the stereotypical flag-1 system as observed in E. coli K-12 str. MG1655 (Fig. 6). However, on the basis of sequence conservation, the Sodalis flag-1 locus is closer to its flag-1 counterpart, with the protein complement of the intact S. praecaptivus HS1 flag-1 locus sharing 59.2\% AAI with the E. coli K-12 str. MG1655 flag-1 locus, while only sharing $47.5 \%$ AAI with the $S$. praecaptivus flag-4 locus. This suggests that, at least in Sodalis, the flag-1 was duplicated in its entirety, prior to sequence divergence of the flag-4 locus.

On the basis of the protein content encoded by the loci and their phylogenetic clustering, it was predicted that these additional flagellar loci code for two peritrichous (flag-3 and flag-4) and polar (flag-5) flagellar systems. A number of studies have shown the co-existence of two flagellar systems, particularly in Vibrio and Aeromonas spp., which host both a polar and lateral flagellar system that facilitate movement in liquid environments and across surfaces, respectively [2]. It can be envisaged that the primary (flag-1) peritrichous and lateral (flag-2) flagellar systems enable similar interchangeable modes of motility in the Enterobacterales. Similarly, the lateral (flag-2) and polar (flag-5) flagellar loci have been shown to facilitate swarming and swimming motility in $P$. shigelloides, respectively [16]. The combination of potentially dual peritrichous flagellar systems (flag-1 and flag3; flag-1 and flag-4), however, is more puzzling. One possibility is that the second copy may serve as a source for spare-parts should the flag-1 locus no longer be functional. This is plausible among the Sodalis spp., where for example, the flag-1 of S. glossinidius 'morsitans B4' and S. pierantonius SOPE have undergone extensive gene decay, deletion and disruption through transposon integration (Fig. 6), although this degeneration is also evident in the flag-4 loci. However, the relatively low sequence conservation between the flag-1 and flag-3 (52.23\% AAI) and the flag-1 and flag-4 (49.69\% AAI) loci proteins suggest they may not be interchangeable. The main flagellar filament component, flagellin, is highly immunogenic and, upon recognition by host receptors, trigger both local and systemic innate and adaptive immune responses against bacteria in both plant and animal hosts [4]. Host-associated bacteria have evolved a number of means to counteract this process, including phase variable expression of flagellins with distinct antigenic properties and posttranslational glycosylation of the flagellin protein. Given that many of the enterobacterial taxa which are predicted to encode two peritrichous flagellar systems originated from animal and plant hosts, it is possible that the flag-1 and flag-3 loci are expressed under distinct conditions. It can also not be excluded that the flag-3 locus-encoded system plays a role other than motility. The flag-1 system of several pathogenic bacteria has, for example, been shown to serve as secretion system for several distinct virulence factors, while the presence of predicted secretion targets in the cargo regions of flag-2 loci may indicate a similar role for this lateral flagellar system [15, 33, 34]. Finally, previous analyses, of the flag-2 loci in particular, have highlighted the prevalence of transposable elements, pseudogenes and en bloc gene deletions within additional flagellar loci $[14,15]$. It is possible that disruptions in the additional flag loci, may have resulted in non-functionality of previously functioning flagellar systems and concomitant energy conservation. It must be noted, however, that while transposase genes, pseudogenes and en bloc deletions are prominent features of the flag-4 loci, they are restricted to a very small number of flag-3a (11/155-7.1\%) and flag-3b (6/95-6.3\%) loci, while all flag-5 loci appear to be intact. Further characterization and knock-out mutagenesis of the additional flagellar systems need to be undertaken to unravel their functionality and biological roles. Furthermore, the cellular placement of the flag-3 and flag-4 loci was predicted purely on the basis of in silico data in this study, and further microscopic evaluation is required to determine their number and cellular localization of the flagella they encode.

\section{Conclusions}

A comprehensive comparative genomic analysis showed that supernumerary flagellar systems represent a relatively common feature among members of the order Enterobacterales with one fifth of the enterobacterial taxa harbouring a lateral (flag-2), predicted peritrichous (flag-3 and flag-4) or polar (flag-5) locus on their genome, in addition to the primary (flag-1) flagellar system. Furthermore, a limited number of enterobacterial taxa incorporate loci coding for three distinct flagellar systems, which has to date not been reported for any bacterium. Considering the energetic burden of maintaining and operating multiple flagellar systems, it is possible that they play important biological roles in the Enterobacterales and may provide competitive advantage to the bacteria that possess them.

\section{Methods}

Identification of additional flagellar systems among the Enterobacterales

The presence of additional flagellar loci was determined on the same dataset of 4028 genomes representative of 
the order Enterobacterales as previously used to study the enterobacterial flag-2 loci [15]. Novel flagellar loci were identified by local tBlastN analyses with the flag- 1 and flag-2 flagellar protein datasets from E. coli K-12 strain MG1655 (NCBI Acc. \# U00096.3; 47 proteins; Flg NMABCDEFGHIKL-FlhEAB-CheZYBRWA-MotAB-Flh DC-FliZACDST-FliEFGHIJKLM NOPQR) and E. coli 042 (NCBI Acc. \# CR753847.1; 38 proteins; LfhAB-LfiR QPNM-LafK-LfiEFGHIJ-LfgNMABDEFGHIJKL-LafWZ ABCDEFSTU) with BioEdit v.7.2.5 [35]. Genomic contigs where Blast hits were obtained were searched upand down-stream to identify the full flag loci. The G + $\mathrm{C} \%$ content of each of the loci was calculated using BioEdit v.7.2.5 [35]. Proteins encoded on the loci were predicted using the Prokaryotic GeneMark.hmm v.2 server [36] and functionally annotated using BlastP analyses against the National Center for Biotechnology Information (NCBI) non-redundant protein sequence database and the Conserved Domain Database using Batch CDSearch [37].

\section{Comparative and phylogenetic analyses}

A representative subset of flag-1 and flag-2 loci, comprising three of each loci per genus in which they occur, was selected for comparative analyses. Orthologous proteins conserved among the representative flag-1 and flag- 2 and the flag-3 to flag-5 loci were identified using Orthofinder 1.1.4 [38]. The 25 orthologous proteins occurring in single copy (SCOs) that were conserved among all the flag loci were utilized to calculate average amino acid identity (AAI) values between and within the different locus groups using compareM v 0.1.0 [39]. The SCOs were furthermore individually aligned using the M-Coffee implementation of T-Coffee [40]. The resultant proteins were concatenated and the alignment was curated using GBlocks v 0.91b [41]. This curated alignment was then used to construct a ML phylogeny with IQTree v 1.6.11 [42] with the appropriate evolutionary model predicted using Modelfinder [43] and phylogeny support through bootstrapping with UFBoot2 $(n=1,000$ replicates) [44]. Similarly, a ML tree was generated on the basis of 45 SCOs conserved among the flag-3a and flag-3b loci. Finally, a ML on the basis of four conserved house-keeping markers, GyrB, InfB, RecA and RpoB was generated as previously described [15] and used to map the prevalence of the novel flag-3, flag-4 and flag-5 loci.

\section{Supplementary information}

Supplementary information accompanies this paper at https://doi.org/10. 1186/s12864-020-07085-w.

Additional file 1: Table S1. Presence/absence of the flag-1 to flag-5 loci among the Enterobacterales. The presence of a specific locus is indicated by the contig/chromosomal sequence NCBI accession number.
The previous nomenclature, isolation source and lifestyle/habitat of the 4028 studied enterobacterial taxa is indicated. Table S2. Average Amino Acid Identity (AAl) values (\%) between the distinct flag loci. The AAl values were calculated on the basis of 25 SCOs conserved among all flag loci using CompareM [39]. A total of three representative taxa were selected for each genus for the flag-1, flag-2 and flag-3 ( $a$ and b) loci to avoid overrepresentation of taxa where the flag loci are particularly prevalent, while all flag-4 and flag-5 loci were included. Table S3. Molecular architectures of the flag-3, flag- 4 and flag-5 loci among the Enterobacterales. The sizes, number of protein coding sequences (CDSs) encoded on the loci, $\mathrm{G}+\mathrm{C}$ contents (\%) and $\mathrm{G}+\mathrm{C}$ deviation (\%) from the average genomic $\mathrm{G}+\mathrm{C}$ content are indicated. Where present, the sizes, locations and number of cargo proteins encoded on variable regions within the loci are shown. Table S4. Functional annotations of the cargo proteins encoded within variable regions in the flag-3 loci. The prevalence of each protein in the flag-3a and flag-3b loci are shown, as are the genera in which each protein is found. The AAI values (\%) between the flag-3 orthologues, the average protein sizes, presence of conserved domains (with e-value and bitscore) and predicted functions are given. Table S5. Functional annotations of the cargo proteins encoded within variable regions in the flag-5 loci. The prevalence of each protein among the flag-5 loci of the distinct Plesiomonas spp. is indicated. The AAl values (\%) between the flag-5 orthologues, the average protein sizes, presence of conserved domains (with e-value and bitscore), top non-Plesiomonas BlastP hits in the NCBI non-redundant protein database (with amino acid identity, e-value and bitscore) and predicted functions are shown.

\section{Abbreviations}

AAl: Amino Acid Identity; CDS: Protein coding sequence; NCBI: National Centre For Biotechnology Information; ML: Maximum Likelihood; SCO: SingleCopy Orthologue

\section{Acknowledgements}

The authors wish to acknowledge the National Research Foundation for student funding (TP).

\section{Authors' contributions}

PDM and TAC conceived the study. PDM and TP performed data analyses and co-wrote the original manuscript. PDM, TP and TAC contributed to the final version. All authors have read and approved the final manuscript.

\section{Authors' information}

PDM (PhD, Dr) is a Senior Lecturer at the School of Molecular \& Cell Biology, University of the Witwatersrand. TP was a post-graduate student who completed her MSc degree in Microbiology and Biotechnology at the University of the Witwatersrand in 2019. TAC (PhD, Prof) is a research leader at the Centre for Microbial Ecology and Genomics, University of Pretoria.

\section{Funding}

TP was funded by the National Research Foundation of South Africa (NRF Masters Scholarship SFH180109299856).

\section{Availability of data and materials}

All data related to this publication are publicly available. The nucleotide and protein sequences from the reference strains for the flag-1 locus (E. coli K-12 strain MG1655), flag-2 locus (E. coli 042) and the two loci which comprise the polar flagellar system of Vibrio parahaemolyticus BB22O are available via the National Centre for Biotechnology Information (NCBI) nucleotide database (http://www.ncbi.nlm.nih.gov/nuccore/) under the accession numbers U00096.3, CR753847.1 and AF069392.3 and U12817.2, respectively. The NCBI nucleotide accession numbers for the contigs/chromosomes on which the target loci were found in the strains analysed in this study are indicated in Additional file 1: Table S1 and Table S3. Similarly, the top protein (BlastP) hits from Additional file 1: Table S5 are available via the NCBI protein database (http://www.ncbi.nlm.nih.gov/protein). The nucleotide as well as predicted protein sequences for the novel flag-3, flag-4 and flag-5 loci (as well as the flag-1 and flag-2 sequences used for comparative purposes) and the phylogenetic data (SCOs, aligned SCOs as well as raw phylogenetic trees) have been made publicly available via Researchgate (https://doi.org/10.13140/RG. 2.2.15622.60482). 


\section{Ethics approval and consent to participate}

Not applicable.

\section{Consent for publication \\ Not applicable.}

\section{Competing interests}

PDM serves as Associate Editor for BMC Genomics. The authors have no other competing interests to declare.

\section{Author details}

${ }^{1}$ School of Molecular \& Cell Biology, University of the Witwatersrand, Wits 2050, South Africa. ${ }^{2}$ Centre for Microbial Ecology and Genomics, University of Pretoria, Pretoria 0002, South Africa.

Received: 24 April 2020 Accepted: 21 September 2020 Published online: 29 September 2020

\section{References}

1. Harshey RM. Bacterial motility on a surface: many ways to a common goal. Ann Rev Microbiol. 2003;57:249-73.

2. Merino S, Shaw JG, Tomás JM. Bacterial lateral flagella: an inducible flagella system. FEMS Microbiol Lett. 2006;263:127-35.

3. Soutourina OA, Bertin PN. Regulation cascade of flagellar expression in gram-negative bacteria. FEMS Microbiol Rev. 2003;27:505-23.

4. Josenhans C, Suerbaum S. The role of motility as a virulence factor in bacteria. Int J Med Microbiol. 2002;291:605-14.

5. Schuhmacher JS, Thormann KM, Bange G. How bacteria maintain location and number of flagellar? FEMS Microbiol Rev. 2015;39:812-22.

6. Liu R, Ochman H. Origins of flagellar gene operons and secondary flagellar systems. J Bacteriol. 2007:189:7098-104.

7. Kim Y-K, McCarter LL. Analysis of the polar flagellar gene system of Vibrio parahaemolyticus. J Bacteriol. 2000;182:3693-704.

8. McCarter LL. Dual flagellar systems enable motility under different circumstances. J Mol Microbiol Biotechnol. 2004;7:18-29.

9. Kirov SM. Bacteria that express lateral flagella enable dissection of the multifunctional roles of flagella in pathogenesis. FEMS Microbiol Lett. 2003; 224:151-9

10. McCarter LL. Polar flagellar motility of the Vibrionaceae. Microbiol Mol Biol Rev. 2001;65:445-62

11. Brenner DJ, Farmer JJ III, Family I. Enterobacteriaceae. In: Brenner DJ, Krieg NR, Staley JT, Garrity GM, Boone DR, Vos P, Goodfellow M, Rainey FA, Schleifer KH, editors. Bergey's Manual of Systematic Bacteriology ${ }^{\circledR}$. 2. Boston: Springer US; 2005. p. 587-607.

12. De Maayer P, Cowan DA. Flashy flagella: flagellin modification is relatively common and highly versatile among the Enterobacteriaceae. BMC Genomics. 2016;17:377

13. Macnab RM. How bacteria assemble flagella. Ann Rev Microbiol. 2003:57:77100

14. Ren CP, Beatson SA, Parkhill J, Pallen MJ. The flag-2 locus, an ancestral gene cluster, is potentially associated with a novel flagellar system from Escherichia coli. J Bacteriol. 2005;187:1430-40.

15. De Maayer P, Pillay T, Coutinho TA. Comparative genomic analysis of the secondary flagellar (flag-2) system in the order Enterobacterales. BMC Genomics. 2020:21:100

16. Merino S, Quilini E, Fulton KM, Twine SM, Tomás JM. The polar and lateral flagella from Plesiomonas shigelloides are glycosylated with legionaminic acid. Front Microbiol. 2015;6:649.

17. Saini S, Koirala S, Floess E, Mears PJ, Chemla YR, Golding I, Aldridge C, Aldridge PD, Rao CV. FliZ induces a kinetic switch in flagellar gene expression. J Bacteriol. 2010;192:6477-81.

18. Logan SM. Flagellar glycosylation - a new component of the motility repertoire? Microbiology. 2006;152:1249-62.

19. Merino S, Tomás JM. Gram-negative flagella glycosylation. Int J Mol Sci. 2014; 15:2840-57.

20. Clarke AJ, Hurtado-Guerrero R, Pathak S, Schuttelkopf AW, Borodkin V Shepherd SM, Birahim AF, van Aalten DM. Structural insights into mechanism and specificity of O-GlcNAc transferase. EMBO J. 2008;27:2780-8.

21. Oakeson KF, Gil R, Clayton AL, Dunn DM, von Neiderhausern AC, Hamil C, et al. Genome degeneration and adaptation in a nascent stage of symbiosis. Genome Biol Evol. 2014;6:76-93.
22. Toh H, Weiss BL, Perkin SAH, Yamashita A, Oshima K, Hattori M, et al. Massive genome erosion and functional adaptations provide insights into the symbiotic lifestyle of Sodalis glossinidius in the tsetse host. Genome Res. 2005;16:149-56.

23. Chari A, Oakeson KF, Enomoto S, Jackson DG, Fisher MA, Dale C. Phenotypic characterization of Sodalis praecaptivus sp. nov., a close non-insectassociated member of the Sodalis-allied lineage of insect endosymbionts. Int J Syst Evol Microbiol. 2015;65:1400-5.

24. Clayton AL, Oakeson KF, Gutin M, Pontes A, Dunn DM, von Niederhausern $A C$, et al. A novel human-infection-derived bacterium provides insights into the evolutionary origins of mutualistic insect-bacterial symbioses. PLoS Genet. 2012;8:e1002990.

25. Rio RVM, Symula RE, Wang J, Lohs C, Wu Y-N, Snyder AK, et al. Insight into the transmission biology and species-specific functional capabilities of tsetse (Diptera: Glossinidae) obligate symbiont Wigglesworthia. mBio. 2012;3: e00240-11.

26. Verbarg S, Frühling A, Cousin S, Brambilla E, Gronow S, Lünsdorf H, et al. Biostraticola tofi gen. Nov. sp. nov., a novel member of the family Enterobacteriaceae. Curr Microbiol. 2008;56:603-8.

27. Echezarreta MA, Klose KE. Vibrio flagellar synthesis. Front Cell Infect Microbiol. 2019;9:131.

28. Zhu S, Nishikino T, Kojima S, Homma M, Liu J. The Vibrio H-ring facilitates the outer membrane penetration of the polar sheathed flagellum. $J$ Bacteriol. 2018:200:e00387-18.

29. De Maayer P, Cowan DA. Comparative genomic analysis of the flagellin glycosylation island of the gram-positive thermophile Geobacillus. BMC Genomics. 2016;17:913

30. Salah Ud-Din AIM, Roujeinikova A. Flagellin glycosylation with pseudaminic acid in Campylobacter and Helicobacter: prospects for development of novel therapeutics. Cell Mol Life Sci. 2017;75:1163-78.

31. Guttenplan SB, Kearns DB. Regulation of flagellar motility during biofilm formation. FEMS Microbiol Rev. 2013;37:849-71.

32. Liu R, Ochman H. Stepwise formation of the bacterial flagellar system. Proc Natl Acad Sci U S A. 2007;104:7116-21.

33. Duan Q, Zhou M, Zhu L, Zhu G. Flagella and bacterial pathogenicity. J Basic Microbiol. 2013:53:1-8

34. Young GM, Schmiel DH, Miller VL. A new pathway for the secretion of virulence factors by bacteria: the flagellar export apparatus functions as a protein-secretion system. Proc Natl Acad Sci U S A. 1999:96:6456-61.

35. Hall TA. BioEdit: a user-friendly biological sequence alignment editor and analysis program for windows 95/98/Nt. Nucl Acids Symp Ser. 1999;41:95-8.

36. Besemer J, Lomsadze A, Borodovsky M. GeneMarkS: a self-training method for prediction of gene starts in microbial genomes. Implications for finding sequence motifs in regulatory regions. Nucleic Acids Res. 2001;29:2607-18.

37. Marchler-Bauer A, Derbyshire MK, Gonzales NR, Lu S, Chitsaz F, Geer LY, et al. CDD: NCBI's conserved domain database. Nucleic Acids Res. 2015:43:222-6.

38. Emms DM, Kelly S. Orthofinder: solving fundamental biases in whole genome comparisons dramatically improves orthogroup inference accuracy. Genome Biol. 2015;16:157.

39. Parks D. Compare M - a toolbox for comparative genomics. 2016; https:// github.com/dparks1134/CompareM.

40. Di Tommaso P, Moretti S, Xenarios I, Orobitg M, Montanyola A, Chang JM, et al. T-coffee: a web server for the multiple sequence alignment of protein and RNA sequences using structural information and homology extension. Nucleic Acids Res. 2011:39:W13-7.

41. Dereeper A, Guignon V, Blanc G, Audic S, Buffet S, Chevenet F, et al. Phylogeny.fr: robust phylogenetic analysis for the non-specialist. Nucleic Acids Res. 2008;36:W465-9.

42. Nguyen LT, Schmidt HA, von Haeseler A, Minh BQ. IQ-TREE: a fast and effective stochastic algorithm for estimating maximum-likelihood phylogenies. Mol Biol Evol. 2015:32:268-74.

43. Kalyaanamoorthy S, Minh BQ, Wong TKF, von Haeseler A, Jermijn LS. ModelFinder: fast model selection for accurate phylogenetic estimates. Nat Methods. 2017:14:587-9.

44. Hoang DT, Chernomor O, von Haeseler A, Minh BQ, Vinh LS. UFBoot2: improving the ultrafast bootstrap approximation. Mol Biol Evol. 2018;35: $518-22$

\section{Publisher's Note}

Springer Nature remains neutral with regard to jurisdictional claims in published maps and institutional affiliations. 